\title{
The Second Love Number of Dark Compact Planets and Neutron Stars with Dark Matter
}

\author{
Yannick Dengler* and Jürgen Schaffner-Bielich ${ }^{\dagger}$ \\ Institut für Theoretische Physik, J. W. Goethe Universität, \\ Max von Laue-Str. 1, 60438 Frankfurt am Main, Germany \\ Laura Tolos \\ Institute of Space Sciences (ICE, CSIC), Campus UAB, \\ Carrer de Can Magrans, 08193 Barcelona, Spain; \\ Institut d'Estudis Espacials de Catalunya (IEEC), 08034 Barcelona, Spain; \\ Faculty of Science and Technology, University of Stavanger, 4036 Stavanger, Norway; \\ Frankfurt Institute for Advanced Studies, Ruth-Moufang-Str. 1, 60438 Frankfurt am Main, Germany
}

(Dated: December 1, 2022)

\begin{abstract}
We study the mass-radius relation and the second Love number of compact objects made of ordinary matter and non-selfannihilating fermionic dark matter for a wide range of dark matter particle masses, and for the cases of weakly and strongly interacting dark matter. We obtain stable configurations of compact objects with radii smaller than $10 \mathrm{~km}$ and masses similar to Earth- or Jupiter-like stellar objects. In certain parameter ranges we find second Love numbers which are markedly different compared to those expected for neutron stars without dark matter. Thus, by obtaining the compactness of these compact objects and measuring their tidal deformability from gravitational wave detections from binary neutron star mergers, the extracted value of second Love number would allow to determine the existence of dark matter inside neutron stars irrespective of the equation of state of ordinary matter.
\end{abstract}

\section{INTRODUCTION}

Astrophysical and cosmological observations indicate that most of the mass of the universe appears in the form of dark matter (DM) 1 3. The nature of DM is however still elusive. Whereas there are direct methods for detecting DM using particle accelerators [4, 5] or analyzing DM scattering off nuclear targets in terrestrial detectors [6], constraints on the properties of DM can be extracted by studying the effects of DM on compact objects, such as white dwarfs and neutron stars. Indeed, the possible gravitational collapse of a neutron star due to accretion of DM can set bounds on the DM properties [7-10]. Also, constraints on DM can be obtained from stars that accrete DM during their lifetime and then collapse into a compact star, inheriting the accumulated DM 11. Moreover, the cooling process of compact objects can be affected by the capture of DM, which subsequently annihilates [12 18. At the same time, self-annihilating DM accreted onto neutron stars may change significantly their kinematical properties 19 or provide a mechanism to seed compact objects with long-lived lumps of strangelets [20. Furthermore, neutron stars that accommodate non-self annihilating DM have emerged as an interesting astrophysical scenario, where to analyze the effects of DM onto hadronic matter (or even quark matter) under extreme conditions [16, 21 39]. In this context, the

\footnotetext{
* dengler@itp.uni-frankfurt.de

$\dagger$ schaffner@astro.uni-frankfurt.de

$\ddagger$ tolos@ice.csic.es
}

existence of compact objects with Earth or Jupiter-like masses but unusual small radii have been put forward [40 42, allowing for a new scenario to determine the existence and nature of DM.

Recently, the detection of gravitational waves (GW) from the merger of a binary neutron star system has opened a new venue for probing the existence of DM [43, 44. Whereas the structure of neutron stars can be modified by DM in the post-merger phase [45 48, the GW signal also depends on the deformation of the binary neutron star system in the inspiral stage. This information is encoded in second Love number and, hence, in the tidal deformability [49, 50. The presence of DM will change the tidal deformability, thus allowing for constraining DM properties. Also, the combination of GW detections with X-ray astronomy, from NICER [51, 52] or eXTP [53, and radio, e.g. SKA [54, will help to determine the presence and nature of DM in compact objects, such as neutron stars.

In this paper we study compact stellar objects that are made of ordinary neutron star matter (OM) admixed with non-self annihilating DM. In order to do so, we have corrected [55, and extended the previous works [40, 41] by analysing the mass and radius configurations of these compact objects for different DM particle masses, and for weakly and strongly interacting DM. Indeed, in the present manuscript we have performed a detailed and deeper analysis of the different configurations, delineating the trends with the mass and the strength of the DM interaction, in particular for the solutions that differ from the neutron-star and white-dwarf branches. Our final goal is to determine the second Love number of these 
new mass-radius configurations in view of the recent results coming from GW events. The tidal deformability has been investigated for boson stars in Refs. [56, 57, for pure fermionic dark stars [58, 59, and for a mixture of bosonic DM and neutron star matter in Refs. 34, 37. Note that Ellis et al. in Ref. 34 mention fermionic DM but the calculations are only done for bosonic DM. There is also one recent work adding fermionic DM in neutron stars to constrain DM parameters from pulsar data 60. However, a calculation of the Love number is missing. In all the above cases, the parameters of DM are confined so that the compact star configurations have masses of the order of ordinary neutron stars. A systematic and parametric study in terms of different DM masses and interactions has not been done in the literature. To the best of our knowledge, there is no work yet studying the second Love numbers as function of compactness for all different possible configurations of mass and radius in the case of fermionic DM admixed with fermionic OM, as discussed in the present manuscript [61].

\section{FORMALISM}

\section{A. TOV-equations} for dark compact stellar objects

In the following, we investigate compact objects that are made of OM admixed with non-self-annihilating DM, following the steps of Ref. 40. These two types of matter are described by two fluids that only interact gravitationally [24. One proceeds by solving simultaneously the coupled TOV equations for $\mathrm{OM}$ and DM in dimensionless form as

$$
\begin{aligned}
& \frac{d p_{O M}^{\prime}}{d r}=-\left(p_{O M}^{\prime}+\varepsilon_{O M}^{\prime}\right) \frac{d \nu}{d r}, \\
& \frac{d m_{O M}}{d r}=4 \pi r^{2} \varepsilon_{O M}^{\prime}, \\
& \frac{d p_{D M}^{\prime}}{d r}=-\left(p_{D M}^{\prime}+\varepsilon_{D M}^{\prime}\right) \frac{d \nu}{d r}, \\
& \frac{d m_{D M}}{d r}=4 \pi r^{2} \varepsilon_{D M}^{\prime}, \\
& \frac{d \nu}{d r}=\frac{\left(m_{O M}+m_{D M}\right)+4 \pi r^{3}\left(p_{O M}^{\prime}+p_{D M}^{\prime}\right)}{r\left(r-2\left(m_{O M}+m_{D M}\right)\right)},
\end{aligned}
$$

where $p^{\prime}$ and $\varepsilon^{\prime}$ are the dimensionless pressure and energy density respectively, defined as $p^{\prime}=\frac{p}{m_{f}^{4}}$ and $\varepsilon^{\prime}=\frac{\varepsilon}{m_{f}^{4}}$, with $m_{f}$ being either the neutron mass $\left(m_{n}\right)$ or the mass of the fermionic DM particle $\left(m_{F}\right)$. We choose the latter one. Then, the physical mass and radius for each specie are $R=\left(M_{p} / m_{F}^{2}\right) r$ and $M=\left(M_{p}^{3} / m_{F}^{2}\right) m$, respectively, where $M_{p}$ is the Planck mass 62.

The EoS for OM is given by the equation-of-state EoSI from [63]. The different EoSs obtained in [63] are constrained by using input from low-energy nuclear physics using chiral effective theory and the high-density limit from perturbative QCD. The regime between these two limits is described by interpolated piecewise polytropes that are restricted by observational pulsar data. In particular, the EoSI is the most compact one with maximum masses of $2 \mathrm{M}_{\odot}$. We, moreover, map EoSI with an inner and outer crust EoS using [64] and 65], respectively. For $\rho<3.3 \times 10^{3} \mathrm{~g} / \mathrm{cm}^{3}$ we use the Harrison-Wheeler EoS 66].

In the case of DM, we consider non-selfannihilating fermions. Two cases are studied, weakly and strongly interacting DM. The strength of the interaction is controlled by the strength parameter, $y=m_{F} / m_{I}$, which is defined as the ratio between the mass of the fermionic dark particle $m_{F}$ and the interaction mass scale $m_{I}$ (see Eqs. (34-35) in Ref. 62]). For strong interactions, $m_{I} \sim 100 \mathrm{MeV}$ (interaction scale related to the exchange of vector mesons) while for weak interactions $m_{I} \sim 300 \mathrm{GeV}$ (exchange of $\mathrm{W}$ and $\mathrm{Z}$ bosons). We consider two extreme cases, that is, $y=10^{-1}$ and $y=10^{3}$ for weakly and strongly interacting DM, respectively.

In order to solve the TOVs one needs to determine the initial central pressure for both species. Following Ref. [40] we fix the ratio of central pressures to different values to take into account different scenarios regarding the impact of the DM component. In particular, in this paper we consider

$$
\mathcal{R}=\frac{p_{0, D M}}{p_{0, O M}}\left(\frac{m_{n}}{m_{F}}\right)^{4},
$$

where $p_{0}$ is the central pressure for DM or OM.

Moreover, for the analysis of the compact objects with DM content, one first has to perform an analysis of the stable configuration for both OM and DM. The stability arguments can be found, for example, in 67, where the stability of the different radial modes in a star is analyzed. In the following we give a short description.

In order to check the stability of a given configuration, one has to consider small radial perturbations of the equilibrium configuration by solving the Sturm-Liouville eigenvalue equation, that yields eigenfrequencies $\omega_{n}$ 67. The eigenfrequencies of the different modes form a discrete hierarchy $\omega_{n}^{2}<\omega_{n+1}^{2}$ with $\mathrm{n}=0,1,2 \ldots$, being real numbers. A negative value of $\omega_{n}^{2}$ leads to an exponential growth of the radial perturbation and collapse of the star. The determination of the sign of the mode results from the analysis of the mass of the star versus the mass density or radius. When the mass reaches an extremum, a mode changes sign. What mode changes sign depends whether the mass-radius relation is going clock- or counterclockwise at the extremum. A clockwise orientation leads to a eigenfrequency going positive again, that is, a mode becoming stable again. A counterclockwise orientation leads to an additional unstable mode. Thus, starting at low mass densities where all eigenfrequencies are positive, one can perform the stability analysis for higher mass densities studying the change of sign of the different modes while keeping the hierarchy among them. Only when all eigenfrequencies are positive, the star will 
be stable 68, 69. In this way, one can study the simultaneous stable regions for OM and DM, as done in Ref. 40].

We close this discussion on the stability of two-fluid configurations in general relativity with a word of caution. While our prescription is valid for one-fluid configurations, see Ref. 68], a full stability analysis for two-fluids would require to solve the coupled Sturm-Liouville eigenvalue equations. More rigorous investigations in this direction just appeared recently involving time-consuming numerical computations 70 72 (for earlier work see [25. 26]). For neutron stars with an admixture of noninteracting DM stable solutions were found in parameter regions which did not seem feasible in a naive analysis 70, 71. The stability of quark stars admixed with DM was studied by solving the combined Sturm-Liouville eigenvalue equations for the two-fluid TOV equations in Ref. [72. Their results shows that only small quark matter masses are dynamically stable leading to stable dark strange planets and dark strangelets, and that the stability of DM stars are mainly affected for small fermion masses. We leave a full stability analysis on the stability of the OM and DM configurations studied here as a topic of investigation for future numerical studies.

\section{B. Tidal Deformability for dark compact stellar objects}

The detection of gravitational waves of binary neutron star mergers, in particular from the GW170817 event 43, has posed recent constraints of the EoS through the so-called tidal deformability during the inspiral phase.

The tidal deformability $\lambda$ measures the induced quadrupole moment, $Q_{i j}$, of a star in response to the tidal field of the companion, $\mathcal{E}_{i j}$ [49, 50]

$$
Q_{i j}=-\lambda \mathcal{E}_{i j}
$$

The tidal deformability is connected to the dimensionless second Love number $k_{2}$ as

$$
\lambda=\frac{2}{3} k_{2} R^{5}
$$

where $R$ is the radius of the star. The tidal Love number can be calculated from

$$
\begin{aligned}
k_{2}= & \frac{8 C^{5}}{5}(1-2 C)^{2}\left[2+2 C\left(y_{R}-1\right)-y_{R}\right] \times \\
& \left\{2 C\left[6-3 y_{R}+3 C\left(5 y_{R}-8\right)\right]+\right. \\
& 4 C^{3}\left[13-11 y_{R}+C\left(3 y_{R}-2\right)+2 C^{2}\left(1+y_{R}\right)\right]+ \\
& \left.3(1-2 C)^{2}\left[2-y_{R}+2 C\left(y_{R}-1\right)\right] \ln (1-2 C)\right\}^{-1},
\end{aligned}
$$

with $C$ being the compactness parameter, that for the case of dark compact objects is given by $C=M / R$, with $M=M_{D M}+M_{O M}$ and $R=R_{\max }\left(R_{O M}, R_{D M}\right)$. The quantity $y_{R} \equiv y(R)$ is obtained by solving, together with the TOVs of Eq. (1), the following differential equation for $y$ 73

$$
r \frac{d y(r)}{d r}+y^{2}(r)+y(r) F(r)+r^{2} Q(r)=0
$$

with

$$
\begin{aligned}
F(r) & =\frac{r-4 \pi r^{3}\left(\left(\varepsilon_{O M}^{\prime}+\varepsilon_{D M}^{\prime}\right)-\left(p_{O M}^{\prime}+p_{D M}^{\prime}\right)\right)}{r-2\left(m_{O M}+m_{D M}\right)} \\
Q(r) & =\frac{4 \pi r}{r-2\left(m_{O M}+m_{D M}\right)} \\
& \times\left[5\left(\varepsilon_{O M}^{\prime}+\varepsilon_{D M}^{\prime}\right)+9\left(p_{O M}^{\prime}+p_{D M}^{\prime}\right)\right. \\
& \left.+\frac{\varepsilon_{O M}^{\prime}+p_{O M}^{\prime}}{c_{s, O M}^{2}}+\frac{\varepsilon_{D M}^{\prime}+p_{D M}^{\prime}}{c_{s, D M}^{2}}-\frac{6}{4 \pi r^{2}}\right] \\
& -4\left[\frac{\left(m_{O M}+m_{D M}\right)+4 \pi r^{3}\left(p_{O M}^{\prime}+p_{D M}^{\prime}\right)}{r^{2}\left(1-\frac{2\left(m_{O M}+m_{D M}\right)}{r}\right)}\right]^{2}
\end{aligned}
$$

where $c_{s}(r)^{2}=d p^{\prime} / d \varepsilon^{\prime}$ is the squared speed of sound for $\mathrm{OM}\left(c_{s, O M}\right)$ and DM $\left(c_{s, D M}\right)$. The starting condition for $y$ in the center of the star is $y(r=0)=2$. Once $k_{2}$ is known, the dimensionless tidal deformability $\Lambda$ can then be determined by the relation

$$
\Lambda=\frac{2 k_{2}}{3 C^{5}} .
$$

\section{RESULTS}

In this section we show our results for the mass-radius relation and second Love number of compact stellar objects with DM content.

Figure 1 shows the stable solutions for compact stellar objects with DM content. We show the total mass of the compact object as a function of the radii of OM (visible matter) for different masses of the dark particle (different rows) and two extreme interaction strengths for the DM component, that is, weakly interacting DM $y=0.1$ (left column) and strongly interacting DM $y=1000$ (right column). In each panel we show the masses and radii for three different ratios between the central pressure of DM with respect to the OM one $(\mathcal{R}$ in Eq. (2)) with red (thin), blue (medium) and grey (thick) lines, respectively. Depending on this ratio, one, two or even three stable branches might appear. Note that we numbered the stable branches with '1', '2' or '3' for the middle value of the ratio (blue lines). Also, note that the present calculation corrects and extends the computation done in Refs. 40, 41] for a larger set of DM particle masses.

For low values of the ratio $\mathcal{R}$ (red lines), the DM content is negligible. Therefore, two stable branches are clearly visible resulting from the dominance of the OM component, that is, the one associated to white dwarfs that appears for radii larger than $10^{3} \mathrm{~km}$ with maximum 


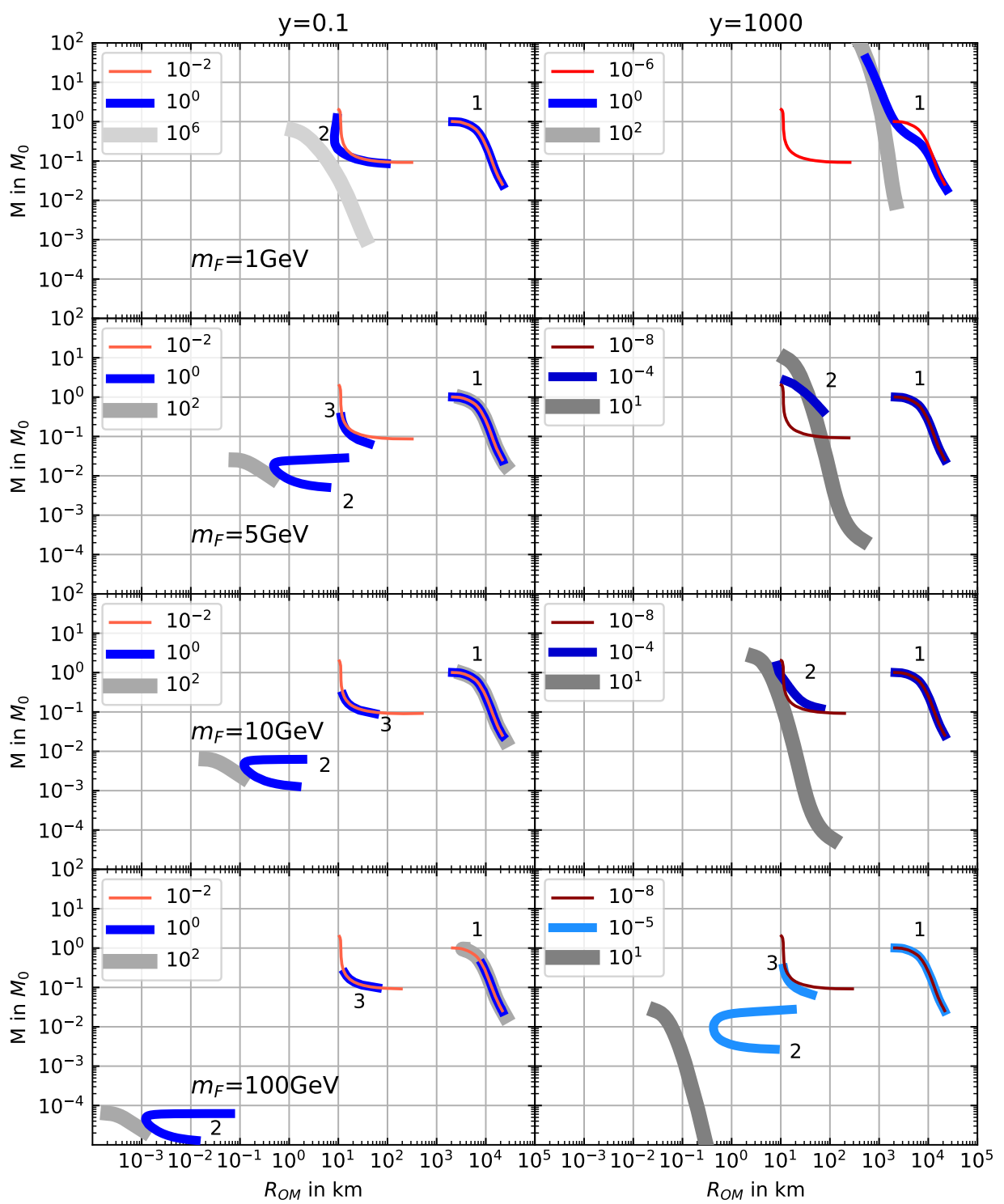

FIG. 1. The total mass as a function of the radius of ordinary matter for compact stellar objects using different DM particle masses (different rows), and for weakly interacting DM (left column) and strongly interacting one (right column).

masses of $1 M_{\odot}$ and the neutron star branch for radii $10-10^{2} \mathrm{~km}$ and maximum masses of $2 M_{\odot}$.

As we increase the ratio $\mathcal{R}$ up to $10^{2}$ (grey lines), whereas we still obtain the white-dwarf branch for the weakly interacting case, for higher central pressures, a new branch appears which is dominated by the DM component. The masses and radii of this branch depend on the mass of the DM particle and the interaction strength. The total mass is dominated by the mass of the DM com- ponent, scaling with the inverse of the squared of the dark particle mass, as discussed in Ref. 62. Moreover, as seen in Ref. 62, for the weakly interacting case, the slope of the mass-radius curve for masses well below the maximum mass is proportional to $R_{D M}^{-3}$. For strongly interacting DM, the mass-radius relation is constant for a given $R_{D M}$, as presented in Ref. 62. Note that this behaviour can not be seen in Fig. 1 as the total mass of the star is plotted against the radius of the OM and not 


\begin{tabular}{|l|l|l|}
\hline$m_{F}[\mathrm{GeV}]$ & WI DM $(\%)$ & SI DM $(\%)$ \\
\hline 1 & 21 & 98.5 \\
\hline 5 & $81(8)$ & 90 \\
\hline 10 & $99(2)$ & 71 \\
\hline 100 & $\sim 100(\sim 0)$ & $81(8)$ \\
\hline
\end{tabular}

TABLE I. Amount of DM mass $\left(M_{D M}\right)$ with respect to the total mass $(M)$ in \% at the maximum mass-radius configuration for the '2' stable branch (labelled with '2' in Fig. 1) for different values of the DM particle $\left(m_{F}\right)$ and for the case of intermediate values of the ratio of pressures between the DM and OM components. We consider weakly-interacting DM (WI DM) and strongly interacting DM (SI DM). Note that for the strongly interacting case with $m_{F}=1 \mathrm{GeV}$ there is only one stable branch with '1'. The number in the brackets corresponds to the amount of DM mass for the '3' stable branch (if it exists), labelled with '3' in Fig. 1 .

the radius of DM.

We now turn our attention to intermediate values of the ratio $\mathcal{R}$ (blue lines) in Fig. 1. For that case, we labelled the different curves in Fig. 1 1 with ' 1 ', '2' or even '3', being ' 1 ' the curve closer to the white-dwarf branch for low values of $\mathcal{R}$. Moreover, we show in Table $\mathbb{I}$ the amount of DM mass with respect to the total mass (in percent) at the maximum mass for '2' in Fig. 1. Note that for the strongly interacting case with $m_{F}=1 \mathrm{GeV}$, there is only one stable branch with ' 1 '. We consider weakly interacting DM (WI DM) and strongly interacting DM (SI DM). The number in the brackets corresponds to the amount of DM mass at the maximum mass for the ' 3 ' stable branch (in case that it exists), labelled with ' 3 ' in Fig. 1 .

We start by analysing the weakly interacting DM and intermediate values of $\mathcal{R}$. For that case and DM particle mass of $1 \mathrm{GeV}$ (close to the nucleon mass), the massradius relation ' 2 ' for $\mathcal{R}=1$ is close to the neutronstar branch of $\mathcal{R}=10^{-2}$, being slightly shifted to lower masses and radii. The amount of DM mass with respect to the total mass at the maximum mass-radius configuration is $21 \%$, as shown in Table II. When the mass of the DM particle is increased, the ' 2 ' branch drops below the neutron-star branch (observed for low values of $\mathcal{R}$ ), and the mass-radius stable configurations appear closer to the DM dominated branch (seen for large values of $\mathcal{R}$ ) and away from the neutron and white-dwarf branches (obtained for low values of $\mathcal{R}$ ). The mass and radius of these solutions, already described as dark compact planets (DCPs) in Ref. [40, scale with the inverse of the DM mass squared. Note that the larger the DM mass is, the larger the central pressure of DM is needed in order to obtain the DCPs (see Eq. (2)). Moreover, the amount of DM for the '2' stable branch increases with increasing DM particle mass, getting closer to the DM dominated branch (observed for large values of $\mathcal{R}$ ), whereas the ' 3 ' branch (when it exists) lies closer to the neutron-star branch (seen for small values of $\mathcal{R}$ ) and, hence, contains a small amount of DM.

For the strongly interacting case and intermediate val- ues of $\mathcal{R}$, the masses and radii of the '2' branch are larger compared to neutron-star branch (seen for low values of $\mathcal{R})$. These solutions for different DM masses contain large amounts of DM, as seen in Table I. Note that for the DM mass of $10 \mathrm{GeV}$, we have a neutron star inside a larger heavy DM halo. Only for a DM mass of $100 \mathrm{GeV}$, the '2' branch drops below the neutron-star branch (observed for low values of $\mathcal{R}$ ). Moreover, for a DM mass of $100 \mathrm{GeV}$, a branch labelled with '3' appears close to the neutron-star branch (obtained for low values of $\mathcal{R}$ ), repeating the pattern seen for the weakly interacting case. This solution contains a small fraction of DM at the maximum mass, as also seen for the ' 3 ' branch in the weakly interacting case.

Next, we analyze the second Love-number $k_{2}$ of the different star configurations shown in Fig. 1. As seen in Eq. (9), a large second Love-number means that the star is deformed easily by an external tidal field (large values of $\Lambda$ ).

Fig. 2 shows $k_{2}$ against the compactness of the star $C$ for the same star configurations displayed in Fig. 1, that is, for different DM masses (different rows) and for weakly interacting DM, $y=10^{-1}$ (left column) and strongly interacting DM, $y=10^{3}$ (right column). Note that the white dwarf branch is not visible in the plots for all cases because the compactness is too low $\left(C \approx 10^{-4}\right)$.

We start by considering low values of $\mathcal{R}$ for all masses and both strengths (red lines). Those are associated to the neutron-star branch, that is, configurations dominated by OM with only a small amount of DM. The values of $k_{2}$ follow the expected pattern for neutron stars with an hadronic core, as described in Ref. [50. By increasing the central pressure, one moves from $k_{2} \rightarrow 0$ at $C \rightarrow 0$ to larger values of $k_{2}$. At a compactness of $C \approx 0.1, k_{2}$ reaches the maximum value and then decreases for large compactness. The maximum compactness of $\approx 0.29$ is achieved by neutron stars with $R \sim 10$ $\mathrm{km}$ with a mass of $2 M_{\odot}$.

Then we study large values of $\mathcal{R}$ for all masses and both strengths (grey lines). As expected, the mass-radius configurations are close to the purely fermionic case 62. The larger the central pressure is, the smaller the $k_{2}$ is with increasing compactness. Moreover, for strongly interacting DM (right column) larger values for $k_{2}$ and $C$ are achieved as compared to the weakly interacting case. Note that the compactness reached for DM dominated stars should be smaller than the limits dictated for pure fermionic matter. Without interactions, this limit is given by $C=0.11$, whereas for strongly interacting matter it results in $C=0.21$ 74.

We finally turn to intermediate values of the ratio $\mathcal{R}$ (blue lines). Starting with the weakly interacting case and a DM particle mass of $1 \mathrm{GeV}$, one notices that the curve follows the trend of the neutron star branch (red line for small values of $\mathcal{R}$ ), as one would expect by the behaviour of branch '2' in the upper left plot of Fig. 1 . However, starting from a fast rise of $k_{2}$ with compactness, two kinks occur at $C \approx 0.012$ and $C \approx 0.07$. Those kinks 


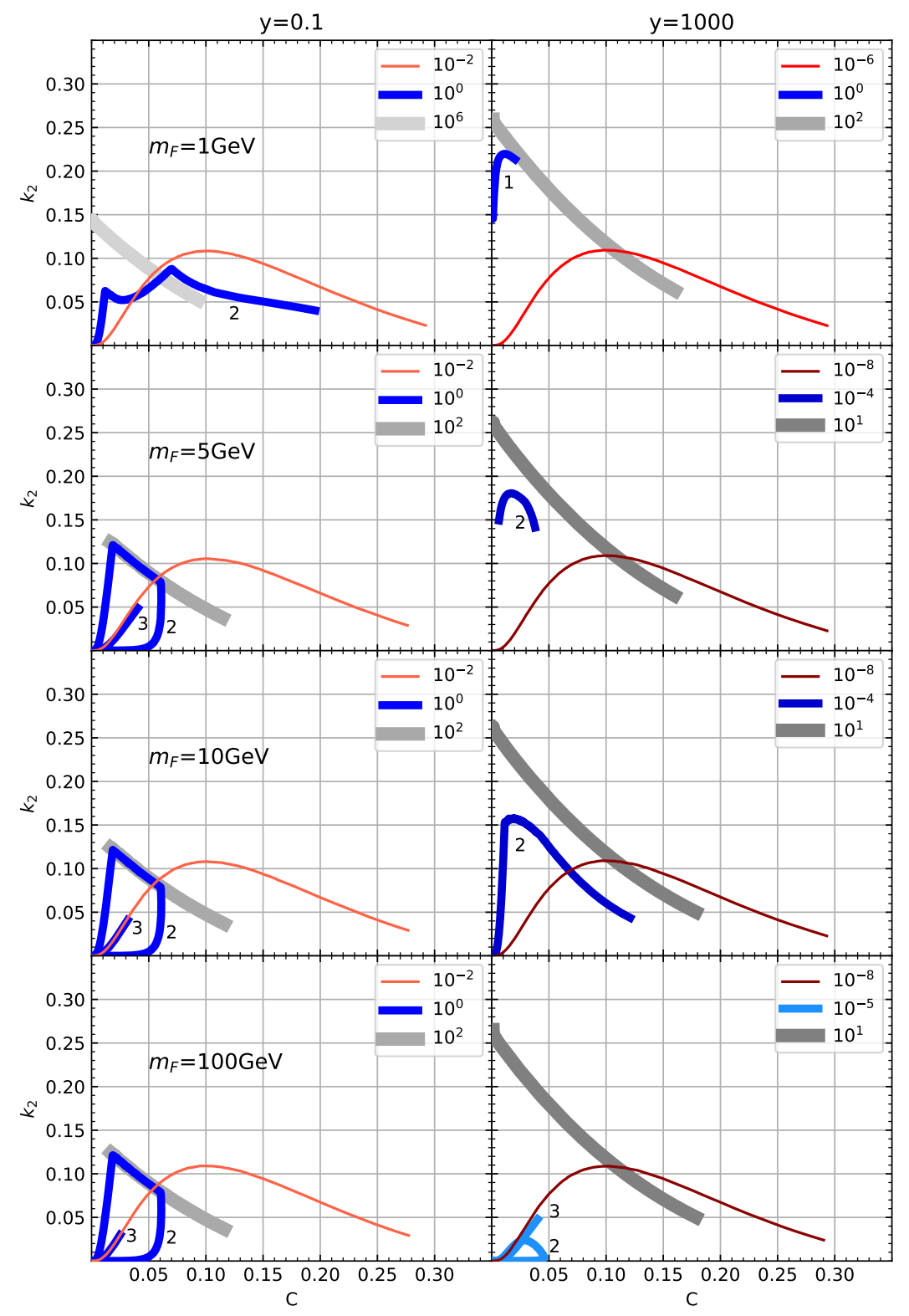

FIG. 2. The second Love number $k_{2}$ as a function of compactness $C$ for compact stellar objects using different DM particle masses (different rows) and for weakly interacting DM (left column) and strongly interacing one (right column).

result from the fact that for the computation of $k_{2}$ one takes the largest radius of a given mass-radius configuration. The largest radius is $R_{O M}$ for small compactness up to the first kink, where $R_{D M}$ becomes the largest, and back to $R_{O M}$ being the largest in the second kink. After the second kink, the values of $k_{2}$ are smaller compared to the OM dominated case. The maximum compactness in this configuration is given by $C \approx 0.2$.

When the DM particle mass is increased to $5 \mathrm{GeV}$ for weakly interacting DM, the behaviour of the $k_{2}$ for intermediate values of the ratio $\mathcal{R}$ (blue lines) changes drastically as compared to the case of a DM particle mass 
of $1 \mathrm{GeV}$. This can be understood by analysing the behaviour of the branches '2' and '3' as compared to the OM dominated case (low values of $\mathcal{R}$ ) or the DM dominated one (large values of $\mathcal{R}$ ) in Fig. 1. With increasing central pressure, $k_{2}$ rises steeply until it reaches the DM dominated mass-radius configuration, displayed by the grey line. A kink occurs as the largest radius moves from $R_{O M}$ to $R_{D M}$. Afterwards, the line follows the DM dominated mass-radius configuration and returns to $C \rightarrow 0$. Then, the branch ' 3 ' appears, being close to the OM dominated mass-radius configuration. This pattern repeats for larger DM particle masses. Note that the values for $k_{2}$ and $C$ are similar for $m_{F}=5,10$ and $100 \mathrm{GeV}$ as both quantities are dimensionless and do not scale with the DM particle mass.

As for the strongly interacting DM matter, the behaviour of the mass-radius configuration for intermediate values of $\mathcal{R}$ changes with increasing DM particle mass, as expected from the behaviour of the mass-radius relation in Fig. 1. For $m_{F}=1 \mathrm{GeV}$ branches '1' and '2' are connected without an unstable regime in between, reaching values for the mass larger than $20 M_{\odot}$ at large radii. As a result, $k_{2}$ reaches $\approx 0.22$, close to the $\mathrm{DM}$ dominated branch for large values of $\mathcal{R}$ (grey line). The maximum compactness is then small, $C \approx 0.02$. For $m_{F}=5$ $\mathrm{GeV}, k_{2}$ first increases up to $C \approx 0.18$ and decreases afterwards. The maximum compactness is reached at $C \approx 0.04$. Note that the values of compactness cannot be extracted directly from Fig. 1, as the OM radius (or visible) radius is plotted there, regardless of the fact that in some mass-radius configuration the largest radius is the $\mathrm{DM}$ one. For $m_{F}=10 \mathrm{GeV}, k_{2}$ first increases steeply up to $C \approx 0.16$ and, then, it decreases following the pattern of the DM dominated case up to a value of $k_{2} \approx 0.04$ at a compactness of $C \approx 0.12$. Finally, for $m_{F}=100 \mathrm{GeV}$, the branches ' 2 ' and ' 3 ' for the strongly interacting DM display a similar behaviour as for the weakly interacting DM case in Fig. 1] although the mass-radius configuration of the DM dominated case (grey line for large values of $\mathcal{R}$ ) is well below the branches ' 2 ' and '3'. Thus, a similar behaviour for $k_{2}$ in the weakly and strongly interacting DM cases is expected, in spite of being the values of $k_{2}$ smaller than those in the DM dominated case. The $k_{2}$ in branch '2' increases up $k_{2} \approx 0.03$. Afterwards it decreases and returns to zero for $C \approx 0.05$. The branch ' 3 ' yields similar values as the $\mathrm{OM}$ dominated case and reaches a compactness of $C \approx 0.04$.

Our results for the neutron-star-like configurations can be compared with previous work on the tidal deformability for neutron stars with an admixture of DM 33, 34. In Ref. 33 it was found that interacting DM fermions in the $\mathrm{MeV}-\mathrm{GeV}$ mass range will form a $\mathrm{DM}$ halo around neutron stars increasing the tidal deformability. We see such an increase in the radius and therefore in the tidal deformability $\Lambda$ for the strongly interacting case and particularly pronounced for low fermion masses. In Ref. 34. the presence of a DM core was leading to a reduced tidal deformability for neutron-star-like configurations for weakly interacting bosons. We find similar configurations with a reduced radius for the weakly interacting case and light fermion masses which would give a reduced tidal deformability $\Lambda$ (see Eq. 9).

As a final remark, we should comment on the different possibilities of formation of compact objects with DM content, that is, by means of the accretion mechanisms of DM onto neutron stars and white dwarfs as well as by the primordial formation of DM clumps surrounded by OM. As argued in Ref. [40 the accretion mechanism (taking into account the local standard DM density) might not explain the quantity of DM obtained inside these compact objects, whereas a larger DM component is expected in the second case. Moreover, there exists the possibility of having dark compact configurations coming from structure formation from DM perturbations growing from primordial overdensities, as shown in Ref. [75]. Initial density perturbations can produce compact objects of DM with masses from substellar masses to masses of several million solar masses. The key input are vector boson interactions of fermionic DM, which allows for creating out of the initial primordial density perturbations compact objects of various mass scales. The larger the interaction, the smaller the compact objects can be. E.g. compact objects of planetary size can be produced for large interaction strengths or by fragmentation or mergers of compact objects. We note that the interaction used in Ref. [49] via vector boson exchange gives the kind of EoS for DM we are using in our investigations.

\section{SUMMARY}

In this paper we have studied compact objects that are made of OM admixed with non-self annihilating DM. We have analysed their masses and radius for different DM particle masses for the weakly and strongly interacting DM. We confirm our previous finding of stable configurations of $\mathrm{OM}$ and $\mathrm{DM}$ with radii smaller than 10 $\mathrm{km}$ and masses similar to Earth- or Jupiter-like stellar objects [40]. Moreover, we have determined the second Love-number of these compact objects in view of the recent first measurements of the tidal deformability from the observation of gravitational waves from neutron star mergers.

We find that the mixture of OM and DM in compact objects leads to the appearance of new mass-radius stable solutions with very distinct second Love numbers. Whereas a compact star containing small amounts of DM is almost indistinguishable from normal neutron stars, a large amount of DM inside neutron stars will lead to compact objects with radii smaller than $10 \mathrm{~km}$ and masses similar to Earth- or Jupiter-like stellar objects. The second Love numbers of these dark compact stellar objects (or dark compact planets) will then be very different from the ones of normal neutron stars. The tidal deformability of these dark compact stellar objects will make them distinguishable and detectable in the merger of compact 
objects with future GW detectors.

In addition, it turns out that for a not too small admixture of DM inside neutron stars, the second Love number is significantly different compared to ordinary neutron stars or strange quark stars without DM. As an example, Love numbers of stars made only of strange quark matter are much higher than those presented here for the same compactness, as shown in Ref. [50. Those are closer to the Buchdahl limit for incompressible stars, and therefore strange quark stars will be distinguishable from the dark compact objects shown in the present manuscript. This feature can serve as an experimentally accessible observable for the presence of DM inside neutron stars for a known compactness and tidal deformability of the merging compact star which does not rely on the EoS of OM. We expect that future improved measurements coming from next-generation gravitational interferome- ters will be able to utilize this observable to assess the DM content of merging compact stars, as emphasized in Refs. [34, 37, 56, 57, 60].

\section{ACKNOWLEDGMENTS}

L.T. acknowledges support from Agencia Estatal Consejo Superior de Investigaciones Científicas project Nr. 202050I008, by PID2019-110165GB-I00 financed by MCIN/AEI/10.13039/501100011033 and by Generalitat Valenciana under contract PROMETEO/2020/023. This research has also been supported by the EU STRONG2020 project under the program H2020-INFRAIA-2018-1 grant agreement no. 824093, by PHAROS COST Action CA16214 and by the CRC-TR 211 'Strong-interaction matter under extreme conditions'- project Nr. 315477589 - TRR 211.
[1] P. A. R. Ade et al. (Planck), Planck 2013 results. XVI. Cosmological parameters, Astron. Astrophys. 571, A16 (2014), arXiv:1303.5076 [astro-ph.CO].

[2] M. Betoule et al. (SDSS), Improved cosmological constraints from a joint analysis of the SDSS-II and SNLS supernova samples, Astron. Astrophys. 568, A22 (2014), arXiv:1401.4064 [astro-ph.CO],

[3] M. Khlopov, What comes after the standard model?, Progress in Particle and Nuclear Physics 116, 103824 (2021)

[4] G. Aad et al. (ATLAS), Search for dark matter candidates and large extra dimensions in events with a jet and missing transverse momentum with the ATLAS detector, JHEP 04, 075, arXiv:1210.4491 [hep-ex]

[5] S. Chatrchyan et al. (CMS), Search for Dark Matter and Large Extra Dimensions in Monojet Events in $p p$ Collisions at $\sqrt{s}=7 \mathrm{TeV}$, JHEP 09, 094, arXiv:1206.5663 [hep-ex]

[6] M. Klasen, M. Pohl, and G. Sigl, Indirect and direct search for dark matter, Prog. Part. Nucl. Phys. 85, 1 (2015), arXiv:1507.03800 [hep-ph],

[7] I. Goldman and S. Nussinov, Weakly Interacting Massive Particles and Neutron Stars, Phys. Rev. D 40, 3221 (1989)

[8] C. Kouvaris and P. Tinyakov, Excluding Light Asymmetric Bosonic Dark Matter, Phys. Rev. Lett. 107, 091301 (2011), arXiv:1104.0382 [astro-ph.CO].

[9] J. Fuller and C. Ott, Dark Matter-induced Collapse of Neutron Stars: A Possible Link Between Fast Radio Bursts and the Missing Pulsar Problem, Mon. Not. Roy. Astron. Soc. 450, L71 (2015), arXiv:1412.6119 [astroph.HE]

[10] J. F. Acevedo, J. Bramante, A. Goodman, J. Kopp, and T. Opferkuch, Dark Matter, Destroyer of Worlds: Neutrino, Thermal, and Existential Signatures from Black Holes in the Sun and Earth, JCAP 04, 026, arXiv:2012.09176 [hep-ph]

[11] C. Kouvaris and P. Tinyakov, Constraining Asymmetric Dark Matter through observations of compact stars,
Phys. Rev. D 83, 083512 (2011), arXiv:1012.2039 [astroph.HE]

[12] C. Kouvaris, WIMP Annihilation and Cooling of Neutron Stars, Phys. Rev. D 77, 023006 (2008), arXiv:0708.2362 [astro-ph]

[13] G. Bertone and M. Fairbairn, Compact Stars as Dark Matter Probes, Phys. Rev. D 77, 043515 (2008), arXiv:0709.1485 [astro-ph]

[14] C. Kouvaris and P. Tinyakov, Can Neutron stars constrain Dark Matter?, Phys. Rev. D 82, 063531 (2010), arXiv:1004.0586 [astro-ph.GA]

[15] M. McCullough and M. Fairbairn, Capture of Inelastic Dark Matter in White Dwarves, Phys. Rev. D 81, 083520 (2010), arXiv:1001.2737 [hep-ph].

[16] A. de Lavallaz and M. Fairbairn, Neutron Stars as Dark Matter Probes, Phys. Rev. D 81, 123521 (2010), arXiv:1004.0629 [astro-ph.GA]

[17] A. Sedrakian, Axion cooling of neutron stars. II. Beyond hadronic axions, Phys. Rev. D 99, 043011 (2019), arXiv:1810.00190 [astro-ph.HE].

[18] S. A. Bhat and A. Paul, Cooling of Dark-Matter Admixed Neutron Stars with density-dependent Equation of State, Eur. Phys. J. C 80, 544 (2020), arXiv:1905.12483 [hep$\mathrm{ph}]$,

[19] M. A. Perez-Garcia and J. Silk, Dark matter seeding and the kinematics and rotation of neutron stars, Phys. Lett. B 711, 6 (2012), arXiv:1111.2275 [astro-ph.CO].

[20] M. A. Perez-Garcia, J. Silk, and J. R. Stone, Dark matter, neutron stars and strange quark matter, Phys. Rev. Lett. 105, 141101 (2010), arXiv:1007.1421 [astro-ph.CO].

[21] S. I. Blinnikov and M. Y. Khlopov, Possible Astronomical Effects of Mirror Particles, Sov. Astron. 27, 371 (1983).

[22] M. Y. Khlopov, G. M. Beskin, N. G. Bochkarev, L. A. Pustilnik, and S. A. Pustilnik, Observational Physics of the Mirror World, Sov. Astron. 35, 21 (1991).

[23] A. Li, F. Huang, and R.-X. Xu, Too massive neutron stars: The role of dark matter?, Astropart. Phys. 37, 70 (2012), arXiv:1208.3722 [astro-ph.SR].

[24] F. Sandin and P. Ciarcelluti, Effects of mirror dark mat- 
ter on neutron stars, Astropart. Phys. 32, 278 (2009), arXiv:0809.2942 [astro-ph]

[25] S. C. Leung, M. C. Chu, and L. M. Lin, Dark-matter admixed neutron stars, Phys. Rev. D 84, 107301 (2011), arXiv:1111.1787 [astro-ph.CO],

[26] S. C. Leung, M. C. Chu, and L. M. Lin, Equilibrium Structure and Radial Oscillations of Dark Matter Admixed Neutron Stars, Phys. Rev. D 85, 103528 (2012), arXiv:1205.1909 [astro-ph.CO],

[27] Q.-F. Xiang, W.-Z. Jiang, D.-R. Zhang, and R.-Y. Yang, Effects of fermionic dark matter on properties of neutron stars, Phys. Rev. C 89, 025803 (2014), arXiv:1305.7354 [astro-ph.SR],

[28] I. Goldman, R. N. Mohapatra, S. Nussinov, D. Rosenbaum, and V. Teplitz, Possible Implications of Asymmetric Fermionic Dark Matter for Neutron Stars, Phys. Lett. B 725, 200 (2013), arXiv:1305.6908 [astro-ph.CO],

[29] M. Khlopov, Fundamental Particle Structure in the Cosmological Dark Matter, Int. J. Mod. Phys. A 28, 1330042 (2013), arXiv:1311.2468 [astro-ph.CO].

[30] P. Mukhopadhyay and J. Schaffner-Bielich, Quark stars admixed with dark matter, Phys. Rev. D 93, 083009 (2016), arXiv:1511.00238 [astro-ph.HE]

[31] Z. Rezaei, Study of Dark-Matter Admixed Neutron Stars using the Equation of State from the Rotational Curves of Galaxies, Astrophys. J. 835, 33 (2017), arXiv:1612.02804 [astro-ph.HE]

[32] G. Panotopoulos and I. Lopes, Dark matter effect on realistic equation of state in neutron stars, Phys. Rev. D 96, 083004 (2017), arXiv:1709.06312 [hep-ph].

[33] A. Nelson, S. Reddy, and D. Zhou, Dark halos around neutron stars and gravitational waves, JCAP 07, 012 . arXiv:1803.03266 [hep-ph]

[34] J. Ellis, G. Hütsi, K. Kannike, L. Marzola, M. Raidal, and V. Vaskonen, Dark Matter Effects On Neutron Star Properties, Phys. Rev. D 97, 123007 (2018), arXiv:1804.01418 [astro-ph.CO],

[35] M. I. Gresham and K. M. Zurek, Asymmetric Dark Stars and Neutron Star Stability, Phys. Rev. D 99, 083008 (2019), arXiv:1809.08254 [astro-ph.CO]

[36] O. Ivanytskyi, V. Sagun, and I. Lopes, Neutron stars: New constraints on asymmetric dark matter, Phys. Rev. D 102, 063028 (2020), arXiv:1910.09925 [astro-ph.HE].

[37] D. R. Karkevandi, S. Shakeri, V. Sagun, and O. Ivanytskyi, Bosonic dark matter in neutron stars and its effect on gravitational wave signal, Phys. Rev. D 105, 023001 (2022), arXiv:2109.03801 [astro-ph.HE]

[38] D. Sen and A. Guha, Implications of feebly interacting dark sector on neutron star properties and constraints from GW170817, Mon. Not. Roy. Astron. Soc. 504, 3 (2021), arXiv:2104.06141 [hep-ph],

[39] A. Guha and D. Sen, Feeble DM-SM interaction via new scalar and vector mediators in rotating neutron stars, JCAP 09, 027, arXiv:2106.10353 [hep-ph],

[40] L. Tolos and J. Schaffner-Bielich, Dark Compact Planets, Phys. Rev. D 92, 123002 (2015), [Erratum: Phys.Rev.D 103, 109901 (2021)], arXiv:1507.08197 [astro-ph.HE]

[41] M. Deliyergiyev, A. Del Popolo, L. Tolos, M. Le Delliou, X. Lee, and F. Burgio, Dark compact objects: an extensive overview, Phys. Rev. D 99, 063015 (2019), arXiv:1903.01183 [gr-qc]

[42] A. Del Popolo, M. Deliyergiyev, M. Le Delliou, L. Tolos, and F. Burgio, On the change of old neutron star masses with galactocentric distance, Phys. Dark Univ.
28, 100484 (2020), arXiv:1904.13060 [gr-qc]

[43] B. P. Abbott et al. (LIGO Scientific, Virgo), GW170817: Observation of Gravitational Waves from a Binary Neutron Star Inspiral, Phys. Rev. Lett. 119, 161101 (2017), arXiv:1710.05832 [gr-qc].

[44] B. P. Abbott et al. (LIGO Scientific, Virgo), GW190425: Observation of a Compact Binary Coalescence with Total Mass $\sim 3.4 M_{\odot}$, Astrophys. J. Lett. 892, L3 (2020), arXiv:2001.01761 [astro-ph.HE]

[45] J. Ellis, A. Hektor, G. Hütsi, K. Kannike, L. Marzola, M. Raidal, and V. Vaskonen, Search for Dark Matter Effects on Gravitational Signals from Neutron Star Mergers, Phys. Lett. B 781, 607 (2018), arXiv:1710.05540 [astro-ph.CO],

[46] M. Bezares, D. Viganò, and C. Palenzuela, Gravitational wave signatures of dark matter cores in binary neutron star mergers by using numerical simulations, Phys. Rev. D 100, 044049 (2019), arXiv:1905.08551 [gr-qc].

[47] C. J. Horowitz and S. Reddy, Gravitational Waves from Compact Dark Objects in Neutron Stars, Phys. Rev. Lett. 122, 071102 (2019), arXiv:1902.04597 [astroph.HE]

[48] A. Bauswein, G. Guo, J.-H. Lien, Y.-H. Lin, and M.-R. $\mathrm{Wu}$, Compact Dark Objects in Neutron Star Mergers, (2020), arXiv:2012.11908 [astro-ph.HE]

[49] T. Hinderer, Tidal Love numbers of neutron stars, Astrophys. J. 677, 1216 (2008), arXiv:0711.2420 [astro-ph].

[50] T. Hinderer, B. D. Lackey, R. N. Lang, and J. S. Read, Tidal deformability of neutron stars with realistic equations of state and their gravitational wave signatures in binary inspiral, Phys. Rev. D 81, 123016 (2010), arXiv:0911.3535 [astro-ph.HE],

[51] M. C. Miller et al., PSR J0030+0451 Mass and Radius from NICER Data and Implications for the Properties of Neutron Star Matter, Astrophys. J. Lett. 887, L24 (2019), arXiv:1912.05705 [astro-ph.HE]

[52] R. Hamburg et al. (Fermi Gamma-ray Burst Monitor Team, LIGO Scientific, Virgo), A Joint Fermi-GBM and LIGO/Virgo Analysis of Compact Binary Mergers From the First and Second Gravitational-wave Observing Runs, Astrophys. J. 893, 100 (2020), arXiv:2001.00923 [astro-ph.HE]

[53] A. L. Watts et al., Dense matter with eXTP, Sci. China Phys. Mech. Astron. 62, 29503 (2019).

[54] A. Watts et al., Probing the neutron star interior and the Equation of State of cold dense matter with the SKA, PoS AASKA14, 043 (2015), arXiv:1501.00042 [astroph.SR],

[55] A numerical error was found in the computation of Ref. [40] and an erratum paper was written.

[56] N. Sennett, T. Hinderer, J. Steinhoff, A. Buonanno, and S. Ossokine, Distinguishing Boson Stars from Black Holes and Neutron Stars from Tidal Interactions in Inspiraling Binary Systems, Phys. Rev. D 96, 024002 (2017), arXiv:1704.08651 [gr-qc].

[57] A. Maselli, P. Pnigouras, N. G. Nielsen, C. Kouvaris, and K. D. Kokkotas, Dark stars: gravitational and electromagnetic observables, Phys. Rev. D 96, 023005 (2017), arXiv:1704.07286 [astro-ph.HE].

[58] A. B. Wahidin and A. Sulaksono, Tidal Love Numbers of Fermionic Dark Stars, J. Phys. Conf. Ser. 1354, 012012 (2019)

[59] A. B. Wahidin, A. Rahmansyah, and A. Sulaksono, Effect of scalar boson on fermionic dark stars, Int. J. Mod. Phys. 
D 28, 1950071 (2019), arXiv:1901.03390 [gr-qc]

[60] F. Di Giovanni, N. Sanchis-Gual, P. Cerdá-Durán, and J. A. Font, Can fermion-boson stars reconcile multimessenger observations of compact stars?, (2021), arXiv:2110.11997 [gr-qc]]

[61] After the submission of the present manuscript, a contribution to the proceedings of PANIC2021 [76] and a special issue to Galaxy journal [77] appeared on tidal deformability of fermionic dark matter admixed with neutron star matter.

[62] G. Narain, J. Schaffner-Bielich, and I. N. Mishustin, Compact stars made of fermionic dark matter, Phys. Rev. D 74, 063003 (2006), arXiv:astro-ph/0605724

[63] A. Kurkela, E. S. Fraga, J. Schaffner-Bielich, and A. Vuorinen, Constraining neutron star matter with Quantum Chromodynamics, Astrophys. J. 789, 127 (2014), arXiv:1402.6618 [astro-ph.HE]

[64] J. Negele and D. Vautherin, Neutron star matter at subnuclear densities, Nuclear Physics A 207, 298 (1973)

[65] S. B. Rüster, M. Hempel, and J. Schaffner-Bielich, Outer crust of nonaccreting cold neutron stars, Phys. Rev. C 73, 035804 (2006).

[66] H. T. W. Wheeler, Gravitation Theory and Gravitational Collapse (Univ of Chicago Pr; First Edition, 1965).

[67] T. Shapiro, Black holes, white dwarfs, and neutron stars : the physics of compact objects (Wiley-VCH, 1983).

[68] J. M. Bardeen, K. S. Thorne, and D. W. Meltzer, A Catalogue of Methods for Studying the Normal Modes of Radial Pulsation of General-Relativistic Stellar Models,
Astrophys. J. 145, 505 (1966).

[69] M. G. Alford, S. P. Harris, and P. S. Sachdeva, On the stability of strange dwarf hybrid stars, The Astrophysical Journal 847, 109 (2017).

[70] B. Kain, Radial oscillations and stability of multiplefluid compact stars, Phys. Rev. D 102, 023001 (2020), arXiv:2007.04311 [gr-qc],

[71] B. Kain, Dark matter admixed neutron stars, Phys. Rev. D 103, 043009 (2021), arXiv:2102.08257 [gr-qc].

[72] J. C. Jiménez and E. S. Fraga, Radial oscillations of quark stars admixed with dark matter, (2021), arXiv:2111.00091 [hep-ph]

[73] S. Postnikov, M. Prakash, and J. M. Lattimer, Tidal Love Numbers of Neutron and Self-Bound Quark Stars, Phys. Rev. D 82, 024016 (2010), arXiv:1004.5098 [astro-ph.SR].

[74] J. Schaffner-Bielich, Compact Star Physics (Cambridge University Press, 2020).

[75] J. H. Chang, D. Egana-Ugrinovic, R. Essig, and C. Kouvaris, Structure Formation and Exotic Compact Objects in a Dissipative Dark Sector, JCAP 03, 036 , arXiv:1812.07000 [hep-ph].

[76] V. Sagun, E. Giangrandi, O. Ivanytskyi, I. Lopes, and K. A. Bugaev, Constraints on the fermionic dark matter from observations of neutron stars, (2021), arXiv:2111.13289 [astro-ph.HE].

[77] H. C. Das, A. Kumar, B. Kumar, and S. K. Patra, Dark Matter Effects on the Compact Star Properties, Galaxies 10, 14 (2022), arXiv:2112.14198 [astro-ph.HE]. 